\title{
Medical Image Analysis (MedIA) using Deep Learning
}

\author{
Priyanka Israni, Maulika S. Patel
}

\begin{abstract}
Medical Image analysis has gained momentum in the research since last ten years. Medical images of different modalities like X-rays, Computed Tomography (CT), Magnetic Resonance Imaging (MRI), Ultrasound etc. are generated with an increase of $15 \%$ to $20 \%$ every year. Medical image analysis requires high processing power and huge memory for storing the medical images, processing them, extracting features for useful information and segment the interested area for analysis. Thus, here comes the role of deep learning which proves to be promising for medical image analysis. The major focus of the paper is on exploring the literature on the broad areas of medical image analysis like Image Classification, Tumor/lesion classification and detection, Organ/Sub-structure Segmentation, Image Registration and Image Construction/ Enhancement using deep learning. Paper also highlights the physiological and medical challenges to be taken care, while analyzing medical images. It also discusses the technical challenges of using deep learning for medical image analysis and its solutions.
\end{abstract}

Keywords: Convolutional Neural Network, MRI, CT-Scan, Transfer learning.

\section{INTRODUCTION}

In medical field, the data is available in the form of images and videos. So, human expertise is required to find the abnormal behavior of the hidden organs in the body. Different medical modalities available are X-rays (Chest), Computed Tomography (CT), Magnetic Resonance Imaging (MRI), Ultrasound, Thermographic, Positron emission tomography (PET) as shown in figure 1 respectively. Each of these modalities are assessed for different purpose like $\mathrm{X}$-rays, CT, MRI gives anatomical data by which fractures of bones can be easily detected while thermographic, PET gives functional data which is used to detect the normal and abnormal functioning of cells and tissues.

Early diagnosis of any disease can increase the survival rate of the patient [1]. Specially, when considering cancer, diagnosis at early stage is very crucial. Due to lack of symptoms at the initial stage, cancer remains undetected and hence results in the complicated stage. Thus, there is a need for automated diagnosis systems which can detect the abnormality at the early stage. Google claims that the trained Artificial Intelligence (AI) model detects diabetic retinopathy which almost gives similar results as that of ophthalmologists. The sensitivity score obtained is more than

Revised Manuscript Received on May 20, 2020.

* Correspondence Author

Ms. Priyanka D. Israni*, Computer Engineering,G H Patel College of Engineering \& Technology, V. V. Nagar, Anand. E-mail: priyankapursani@gmail.com

Dr. Maulika S. Patel, Computer Engineering, G H Patel College of Engineering \& Technology, V. V. Nagar, Anand. E-mail: maulika.sandip@gmail.com the experts [26].
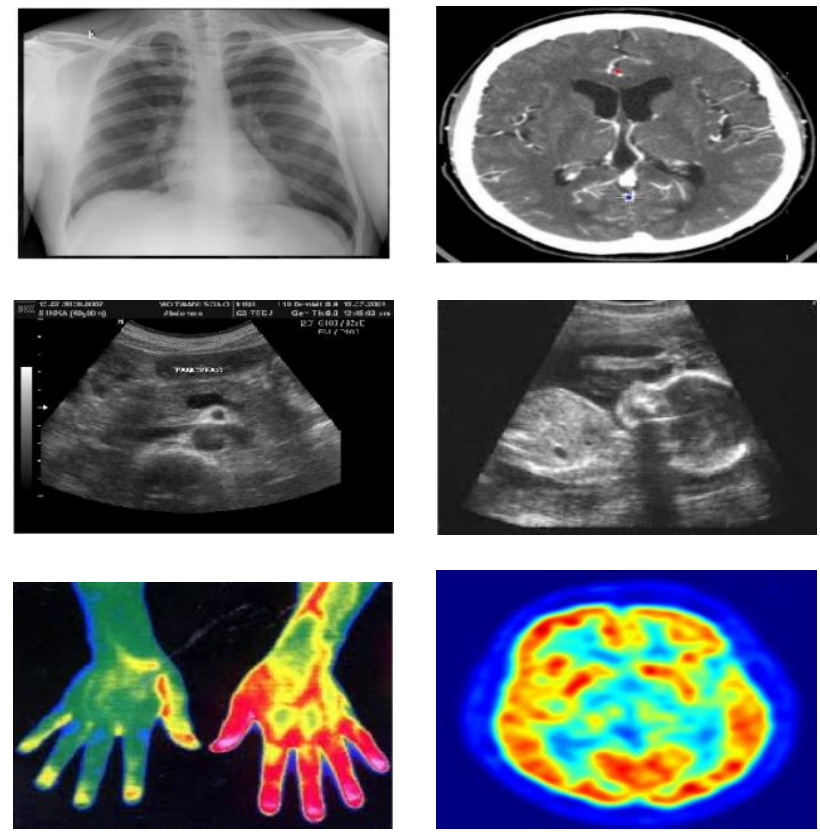

Fig.1. Types of medical images.

Medical Image Analysis has started from last 35 years but amplified since 2000 when machine learning come into practice. The history of medical image analysis is given by $\mathrm{J}$. S. Duncan and N. Ayache [2]. The table 1 shows the history of the medical image analysis.

As there is tremendous data to be analyzed from 2010, deep learning is used for medical image analysis. Currently, transfer learning i.e. using pre-trained models like inception [4-5], [7-8], VGG, etc. for training the data is widely used and gives excellent results.

Section 2 discusses the literature survey on broad areas of medical image analysis and literature analysis. Section 3 discusses the physiological and medical challenges faced while analyzing medical images. Technical challenges while using deep learning are discussed in section 3 along with their solutions.

Table I. History of Medical Image Analysis [2].

\begin{tabular}{|l|l|l|}
\hline $\begin{array}{c}\text { Sr. } \\
\text { No. }\end{array}$ & Era & \multicolumn{1}{c|}{ Approaches used } \\
\hline 1. & Pre 1980-1984 & $\begin{array}{l}\text { Pattern Recognition Analysis using } \\
\text { 2D images. }\end{array}$ \\
\hline 2. & $1985-1991$ & $\begin{array}{l}\text { Knowledge based Approaches like } \\
\text { rule-based analysis and rule-based } \\
\text { influencing where used. }\end{array}$ \\
\hline
\end{tabular}




\begin{tabular}{|l|l|l|}
\hline 3. & $1992-1998$ & $\begin{array}{l}\text { Imaging has gained momentum } \\
\text { and integrated analysis on 3D } \\
\text { images has started for the first time. }\end{array}$ \\
\hline 4. & $1999-2010$ & $\begin{array}{l}\text { Machine Learning was introduced } \\
\text { with Shallow Reasoning. }\end{array}$ \\
\hline 5. & 2010- till date & $\begin{array}{l}\text { Machine Learning with Complex } \\
\text { Reasoning by using deep neural } \\
\text { networks. }\end{array}$ \\
\hline
\end{tabular}

\section{LITERATURE SURVEY AND ANALYSIS}

The broad areas of medical image analysis are Image Classification, Tumor/lesion detection and classification, Organ/substructure segmentation, Image Registration and Image Construction/ Enhancement as shown in figure 2.

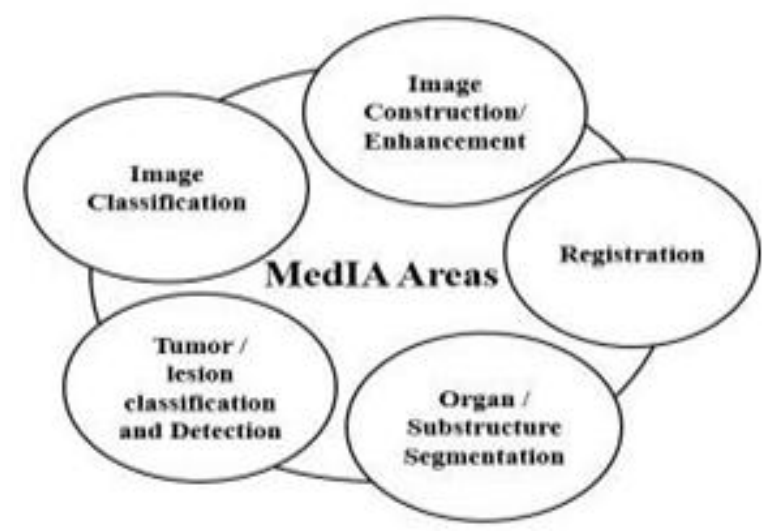

Fig. 2. MedIA Areas

\section{A. Image Classification}

Image Classification is classifying the images into various categories depending upon, whether the image is of the fundus images for the detection of diabetic retinopathy and diabetic macular edema. The images are obtained from EyePACS in the United States and Aravind Eye Hospital, Sankara Nethralaya, and Narayana Nethralaya in India. The authors used an optimized deep convolution neural network trained on the inception-v3 architecture along with distributed stochastic gradient descent for optimization. For better training, the authors used batch normalization and pre-initialization using the weights from the same trained network. A sensitivity of $90.1 \%$ and Specificity of $98.2 \%$ for moderate or worse diabetic retinopathy were obtained.

Esteva et al. [5] used open-access dermatology repositories, the ISIC Dermoscopic Archive, the annotated images from Edinburgh Dermofit Library as malignant and benign, and data from the Stanford Hospital annotated with a specific disease to classify skin cancer. Authors have used transfer learning to train their 129,450 clinical images which can be classified into 2,032 different diseases. The model used is Inception-v3 for training their dataset and 9-fold cross-validation is used for generalized model. The accuracy achieved by the trained CNN model with cross-validation was $55.4 \%$ while accuracy achieved on the same dataset by two dermatologists was $53.3 \%$ and $55.0 \%$.

Payan \& Montana [7] and Hosseini-Asl et al. [8] used Alzheimer's Disease Neuroimaging Initiative (ADNI) dataset which consist MRI images of 755 patients having 2,265 historical scans to classify the images into three classes patient having a disease or not. Gulshan et al. [4] used retinal

as Alzheimer's Disease (AD), Mild Cognitive Impairment (MDI) and Normal Control (NC). Payan \& Montana used 2D and 3D CNN. The accuracy achieved to classify three classes was $85.53 \%$ for $2 \mathrm{D}$ and $89.47 \%$ for 3D. Hosseini-Asl et al. have trained the dataset by using 3D CNN pre-trained on the 3D three stacked autoencoders.

\section{B. Tumour /lesion classification and detection.}

Pan et al. [9] worked on the MRI images of Brain Tumor Segmentation (BRATS 2014) dataset for the detection of brain tumors and its classification. The dataset consists of MRI images of 231 patients. Authors implemented the model by using backpropagation Neural Network, CNN for different kernel sizes and the different number of kernels on $\mathrm{CNN}$. The one-layer CNN structure gives better performance over both sensitivity and specificity with an intersected value of 0.67. Dou et al. [11], proposed the model for the detection of Cerebral Microbleeds from the dataset of $320 \mathrm{MR}$ scans. The authors constructed a 3D fully convolutional network model for the implementation. The Sensitivity and False Positives achieved were $98.29 \%$ and 282.8 respectively.

Kawahara et al. [12] proposed the model for unknown skin lesion detection and classification. The Dermofit Image Library dataset consists of 1300 skin images with 10 class labels. Authors used AlexNet, the pre-trained CNN model to extract the feature and used logistic regression for the classification of 10 class, 5 class and 2 class. The prediction accuracy recorded in 10 class, 5 class, and 2 class were 81.6 $\%, 85.8 \%$, and $94.8 \%$ respectively.

Humpire -Mamani GE et al. [13] used 884 images of thorax-abdomen CT scans and proposed the method for locating multiple organs simultaneously in the images. The authors used a sigmoid layer for multilabel classification with $\mathrm{CNN}$ to train their dataset and to predict the location of 11 structures in the orthogonal view of the images. The performance is measured by calculating the wall distance to the referencing bounding box.

\section{Organ / Substructure Segmentation}

Segmentation is the process of identifying the common characteristics in the pixels of the image and grouping them together to separate Region of Interest (ROI) in the image. S. Pereira et al. [14] M. Havaei et al. [16] and X. Zhao et al. [17] proposed the CNN model for the automatic brain tumor segmentation by using publicly available dataset BRATS. S. Pereira et al. used BRATS13 and BRATS15 dataset and developed the model by using $\mathrm{CNN}$ with the small $3 \times 3$ kernel. The Dice Similarity Coefficient metric of the model for the complete, core, and enhancing regions in BRATS13 is $0.88,0.83,0.77$ respectively and for BRATS15 is $0.78,0.65$ and 0.75 respectively. M. Havaei et al.

[16] proposed the cascaded CNN network in which the output of one $\mathrm{CNN}$ is the supplementary input for the succeeding $\mathrm{CNN}$ and to handle the imbalance tumor labels, 2-phase training procedure is adopted. The dice Similarity Coefficient metric recorded for their model were $0.81,0.72$ and 0.58 for the complete, core, and enhancing regions respectively in BRATS13 dataset. X. Zhao et al. [17] developed a model by assimilating Fully CNNs and Conditional Random Fields (CRFs) on BRATS13, BraTS15, and BRATS16. Instead of using CRFs as the preprocessing step, authors have used CRF- 
RNN which implements CRFs [29] so that training can be done together as a single model. The process of training is done in 3 steps i.e. first, image patches of equal size of each class are used to train FCNNs and by keeping the parameters of FCNNs fixed, the same image slices are used to train CRF-RNN. Finally, image slices are used to fine-tune the complete model. The Dice Similarity Coefficient metric of $0.80,0.66$ and 0.57 on BRATS15 for complete tumor, core tumor, and enhancing tumor respectively were obtained.

Michiel Kallenberg et al. [15] performed breast density segmentation on three clinical datasets. The authors proposed an unsupervised feature learning method by using convolutional sparse autoencoders and recorded the average Area under curve (AUC) as 0.61 .

\section{Image Registration}

Image Registration is aligning two images into one coordinate system for comparison. Cheng et al.

[18] proposed the model to pre-train the deep neural network by using stacked denoising autoencoder for the similarity learning for multiple modality medical images. They used the computed tomography (CT) and MR head image slices from the same patient. The performance is evaluated by using the similarity parameters like mutual information and local cross-correlation.

Simonovsky et al. [19] proposed a CNN model that gives the similarity metric for 3D multiple modalities. The dataset used is publicly available brain atlases Alberts which consist of T1-weighted and T2-weighted MRI scans of 20 infants. Each pair image is aligned and annotated with a mapping of50 anatomical i.e. structural regions. The similarity measure is evaluated by Dice and Jaccard coefficients. The authors obtained the dice and Jaccard coefficient after registration as $0.704 \pm 0.037$ and $0.556 \pm 0.041$ respectively.

Miao et al. [20] proposed the model which extracts the information from the digital reconstructed medical images like radiographs, CT-scans and X-rays. The authors used $\mathrm{CNN}$ regressors to predict the transformation parameters for 2D/3D images for similarity measurement. The authors introduced a feature extraction step to find the 3D complex features that are providing sensitive information for the variables to be regressed by $\mathrm{CNN}$ and Graphical Processing Units (GPUs) are used for better performance.

\section{E. Image Construction/Enhancement}

Image Enhancement is changing the pixels values in the images so that the enhanced images obtained are suitable for analysis. Li et al. [21] proposed the $\mathrm{CNN}$ model for the multi-modality medical images where authors are constructing the Positron Emission Tomography (PET) image from the input MRI image for brain disease diagnosis. Alzheimer's Disease Neuroimaging Initiative (ADNI) database is used which consists of data of 830 patients, where 432 patients have only MRI images while 398 patients have MRI as well as PET images. The authors trained the 398 subjects on 3D CNN for both PET and MRI images and the trained model is used for constructing the PET images for the remaining 432 patients. The classification accuracy on all 830 patients was reported as $0.7621 \pm 0.0205$ and 0.7305

\pm 0.0315 for the constructed and the true PET images respectively.

Bahrami et al. [22] proposed the deep CNN model for constructing 7T MRI images from 3T MRI images by using the anatomical data and intensity i.e. appearance features as inputs which gives the intensity of center voxel in the corresponding $7 \mathrm{~T}$ patch. The authors apply the proposed CNN model to map each input 3T patch to the 7T image patch of 15 patients, each with both 3T and 7T MRI images. The performance of the model is evaluated by the appearance of the constructed 7T MRI image.

\section{F. Literature Analysis}

MedIA using deep learning has increased from 2010 with the major research in segmentation, detection of objects and disease classification, by using microscopic images for the detection of abnormalities in blood and brain image analysis with MRI images for the detection of tumors or

Alzheimer's disease. Convolutional Neural network $(\mathrm{CNN})$, autoencoders and Deep belief Networks (DBNs) are used for training the data. The Emergent learning method adopted is transfer learning i.e. training the data with the pre-trained models like Google's v3 inception which is trained on ImageNet dataset. Transfer Learning with fine-tuning the parameters gives good results as compared to other machine learning and deep learning algorithms [4-5], [7-8].

\section{DISCUSSION AND CHALLENGES}

The development of the global Computer-Aided Diagnosis (CAD) model for medical analysis is still a big challenge as there is a need to focus on the important factors while dealing with human anatomy. As each patient has a different physiological structure, the medical images may contain the variations in the same body part. For example, if the patient is fat the X-ray image of the hidden parts of the body may be different than the thin patient. Even there is inter and intra organ variability due to body development and age. For example, during the embryotic and fetal stages, the growth of the fetus changes in few weeks and same changes may not be recorded in each fetus in same gestational age. Thus, to develop the global automated model age, body development of the patient needs to be considered for the accuracy.

Even considering the physiological challenges is not sufficient to develop the automated models. While implementing the model using deep learning, there are technical issues like the lack of annotated data for the medical images which is required for the verification. The creation of manual annotations from the experts is a challenging task as it requires expert knowledge and extensive labor work, thus becoming the bottleneck for the development of the automated model.

Another problem is to train the model with a smaller data size. By using transfer learning, in which the pre-trained model trained on the data from a source domain where the large dataset of annotated images is available can be used to train the complex model. By, fine-tuning the parameters with data of the target domain can increase the performance on the smaller datasets. Another solution is to use deep learning models as the feature extractors and then use other machine learning or deep learning techniques for training on the extracted features. Also, to enlarge the dataset, Generative Adversarial Networks (GANs) [31] which is a synthetic data generator can be used. GAN's framework has the ability to mimic the behavior of the images and generate synthetic images similar to any domain. It is getting huge important by the computer vision community.

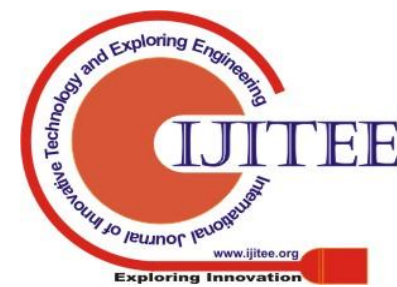




\section{Medical Image Analysis (MedIA) using Deep Learning}

There are various medical modalities and each modality has a different way of assessment i.e. X-rays and CT-scans are used for accessing dysfunctions of anatomy while functional MRI, PET is used for functional assessment. Thus, using both the modalities together can give both anatomical data as well as functional data. A computer-aided diagnosis model which combines the features of multiple modalities will give good results as compared to a single modality.

\section{CONCLUSION}

Artificial Intelligence is playing a crucial role in molding the world towards using computer- aided design models for learning digitally and examining diseases. Based on today's requirement, there is a need for developing generalized automated models that embed the physiological structure of all the developed stages inherited by the human body as well as considers the multiple modalities for examining the diseases. Thus, the research on broad areas of medical image analysis using deep learning is carried out and it proves that the state of art deep learning algorithms detects abnormalities accurately. The future scope includes creating a generalized model for abnormality detection by solving the highlighted physiological, medical and technical issues.

\section{REFERENCES}

1. "Breast cancer survival statistics", Cancer Research UK. (https://www.cancerresearchuk.org/healthprofessional/cancer-statistics/statistics-by-cancer-type/breast-cancer) assessed on 9th January 2020.

2. J. S. Duncan and N. Ayache, "Medical Image Analysis: Progress over Two Decades and the Challenges Ahead", IEEE Transactions on Pattern Analysis and Machine Intelligence, Vol. 22, No. 1, pp. 85-106, 2000.

3. T. Brosch and R. Tam, "Manifold learning of brain MRIs by deep learning", in the proceedings of the Medical Image Computing and Computer-Assisted Intervention, in Lecture Notes in Computer Science, pp. 633-640, 2013, doi: 10.1007/ 978- 3- 642- 40763- 5 _ 78.

4. V. Gulshan, L. Peng, M. Coram, M. C. Stumpe, D. Wu, A Narayanaswamy, S. Venu- gopalan, K. Widner,T. Madams, J. Cuadros, R. Kim, R. Raman, P. C. Nelson, J. L. Mega and D. R. Webster, "Development and validation of a deep learning algorithm for detection of diabetic retinopathy in retinal fundus photographs", Journal of American Medical Association, pp.2402-2410, 2016. doi: 10.1001/jama.2016.17216

5. A. Esteva, B. Kuprel, R.A Novoa, J. Ko, S. M. Swetter, H. M. Blau, S. Thrun, "Dermatologist-level classification of skin cancer with deep neural networks", Nature, pp. 115-118, 2017. doi $10.1038 /$ nature 21056

6. A. Menegola, M. Fornaciali, R. Pires, S. Avila and E. Valle, "Towards automated melanoma screening: exploring transfer learning schemes". arXiv:1609.01228,2016.

7. A. Payan and G. Montana, "Predicting Alzheimer's disease: a neuroimaging study with 3D convolutional neural networks", arxiv: 1502.02506, 2015.

8. E. Hosseini-Asl, G. Gimel' farb and A. El-Baz, "Alzheimer's disease diagnostics by a deeply supervised adaptable 3D convolutional network. arXiv: $1607.00556,2016$.

9. Y. Pan, W. Huang,Z. Lin, W. Zhu, J. Zhou, J. Wong, Z. Ding, "Brain tumor grading based on neural networks and convolutional neural networks", in proceedings of the IEEE Engineering in Medicine and Biology Society, pp. 699-702, 2015. doi 10.1109/EMBC.2015.7318458 .

10. Q. Dou, H. Chen, L. Yu, L. Shi, D. Wang, V. C. Mok, P. A. Heng, “ Automatic cerebral microbleeds detection from MR images via independent subspace anal- ysis based hierarchical features", in Proceedings of the IEEE Engineering in Medicine and Biology Society, pp. 7933-7936, 2015. doi: 10.1109/EMBC.2015.7320232 .

11. Q. Dou,H. Chen,L. Yu, L. Zhao, J. Qin, D. Wang, V.C. Mok, L. Shi ,P.A. Heng, "Automatic detection of cerebral microbleeds from MR images via 3D convolutional neural networks", IEEE Transaction of Medical Imaging, Vol.35, pp.1182-1195, 2016b

12. J. Kawahara, A. BenTaieb and G. Hamarneh, "Deep features to classify skin lesions.", in the proceedings of the IEEE International Symposium on Biomedical Imaging

pp.

$1397-1400$.

doi:

10.1109/ISBI.2016.7493528

13. G. E. Humpire-Mamani, A. A. Setio, B. van Ginneken and C. Jacobs, "Efficient organ localization using multi-label convolutional neural networks in thorax-abdomen CT scans", Journal of Physics in Medicine \& Biology, Vol. 63, No.8, 2018.

14. S. Pereira, A. Pinto, V. Alves, V. and C. A. Silva, "Brain Tumor Segmentation Using Convolutional Neural Networks in MRI Images", IEEE Transactions on Medical Imaging, Vol.35, No.5, pp.1240-1251, 2016.

15. M. Kallenberg, K. Petersen, M. Nielsen, A. Y. Ng, P. Diao, C. Igel and M. Lillholm"Unsupervised deep learning applied to breast density segmentation and mammographic risk scoring", IEEE transactions on medical imaging, Vol.35, No.5, pp.1322-1331,2016.

16. M. Havaei, A. Davy, D. Warde-Farley, A. Biard, A. Courville, Y. Bengio, C. Pal, P. M. Jodoin, and H. Larochelle, "Brain tumor segmentation with deep neural networks.", Medical Image Analysis, Vol.35, pp.18- 31, 2017.

17. X. Zhao, Y. Wu, G. Song, Z. Li, Y. Zhang and Y. Fan, "A deep learning model integrating FCNNs and CRFs for brain tumor segmentation.”, Medical Image Analysis, Vol.43, pp.98-111, 2018.

18. X. Cheng, L. Zhang and Y. Zheng, "Deep similarity learning for multimodal medical images.", Computer Methods in Biomechanics and Biomedical Engineering: Imaging \& Visualization, Vol.6, No.3, pp.248-52 , 2018, doi: 10.1080/ 21681163.2015.1135299

19. M. Simonovsky, B. Gutiérrez-Becker, D. Mateus, N. Navab and N. Komodakis, "A deep metric for multimodal registration." in proceedings of the Medical Image Computing and Computer-Assisted Intervention. In: Lecture Notes in Computer Science, pp. 10-18, 2016, doi: 10.1007/978- 3- 319-46726-9_2.

20. S. Miao, Z. J. Wang and R. Liao, “ A CNN regression approach for real-time 2D/3D registration." IEEE Transactions of Medical Imaging, vol.35, no.5, pp.1352-1363,2016, doi: 10.1109/TMI.2016. 2521800.

21. R. Li, W. Zhang, H. I. Suk, L. Wang, J. Li, D. Shen and S. Ji, "Deep learning based imaging data completion for improved brain disease diagnosis", in proceedings of the Medical Image Computing and Computer- Assisted Intervention. In: Lecture Notes in Computer Science, pp. 305-312, 2014.

22. K. Bahrami, F. Shi, I. Rekik and D. Shen, "Convolutional neural network for re- construction of 7T-like images from 3T MRI using appearance and anatomical features." in proceedings of the Deep Learning in Medical Image Analysis (DLMIA). In: Lecture Notes in Computer Science, 100 08, pp. 39-47, 2016, doi: 10.1007/ 978- 3319- 46976- 8 - 5.

23. V. Sevetlidis, M. V. Giuffrida and S. A. Tsaftaris, "Whole image synthesis using a deep encoder-decoder network", in proceedings of the Simulation and Synthesis in Medical Imaging. In: Lecture Notes in Computer Science, pp. 127-137, 2016, doi: 10.1007/978- 3- 31946630- 9 _ 13

24. A. Y. Hannun, P. Rajpurkar, M. Haghpanahi, G. H. Tison, C. Bourn, M. P. Turakhia and A. Y. Ng, "Cardiologist-level arrhythmia detection and classification in ambulatory electrocardiograms using a deep neural network", Nature medicine, Vol.25, No.1, 2019.

25. P. Rajpurkar, J. Irvin, K. Zhu, B. Yang, H. Mehta, T. Duan, D. Ding, A Bagul, C. Langlotz, K. Shpanskaya and M. P. Lungren, "Chexnet: Radiologist-level pneumonia detection on chest $\mathrm{x}$-rays with deep learning.", arXiv preprint arXiv:1711.05225, 2017.

26. "Google researchers find trained AI detects diabetic retinopathy on par with experts", (https://www.mobihealthnews.com/content/google-researchers-find-tr ained-ai-detects-diabetic-retinopathy- par-experts) assessed on 9th January 2020

27. G. Litjens, T. Kooi, B. E. Bejnordi, A. A. Setio, F. Ciompi, M Ghafoorian, J. A. Van Der Laak, B. Van Ginneken and C. I. Sánchez, "A survey on deep learning in medical image analysis.", Medical Image Analysis, Vol.42, pp.60-88, 2017.

28. A. Janowczyk and A. Madabhushi, "Deep learning for digital pathology image analysis: A comprehensive tutorial with selected use cases.", Journal of pathology informatics 7, 2016.

29. S. Zheng, S. Jayasumana, B. Romera-Paredes, V. Vineet, Z. Su, D. Du, C. Huang and P. H. Torr, "Conditional random fields as recurrent neural networks.", in Proceedings of the IEEE International Conference on Computer Vision, pp. 1529-1537, 2015

30. P. Israni, "Breast Cancer Diagnosis (BCD) Model Using Machine Learning", International Journal of Innovative Technology and Exploring Engineering (IJITEE), vol.8, no.10, pp.4456-4463,2019.

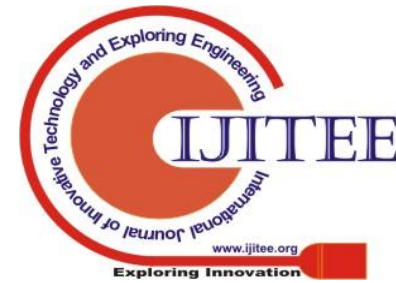


31. I. Goodfellow, J. Pouget-Abadie, M. Mirza, B. Xu, D. Warde-Farley, S. Ozair, A. Courville and Y. Bengio, "Generative adversarial nets," Advances in neural information processing systems, pp. 2672-2680, 2014.

32. R. C. Gonzalez and R. E. Woods, R.E., "Digital image processing", 2002

\section{AUTHORS PROFILE}

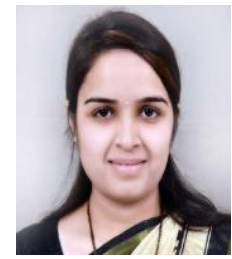

Ms. Priyanka Israni is Assistant Professor at G H Patel College of Engineering \&Technology from last five years. She has seven years of teaching experience. She has published 12 Research papers in various journals and conference on Mobile Computing, Object Oriented Databases, Machine Learning, Medical Data Analysis etc.

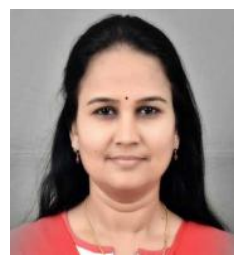

Dr. Maulika S Patel, Professor \& Head of the Computer Engineering Department is associated with G H Patel College of Engineering \& Technology, Vallabh Vidyanagar since last 20 years. She is serving as the head of the department since July 2008. She is awarded a "Certificate of Exemplary performance" and a cash prize for her contributions towards the development of the institute by Charutar Vidya Mandal in March 2007. She has delivered around 20 technical talks at various institutions on topics such as computational biology, neural networks, machine learning etc. She is a member of Computer Society of India, Life member of Indian Society of Technical Education and Fellow of Institution of Engineers, India. She is the Advisor of the GCET CSI-Student Branch. She has been on the reviewer panel for several reputed journals namely IEEE transactions in Computational Biology and Bioinformatics, Applied Soft Computing, Elsevier, Cellular Physiology and Biochemistry, Karger Publishers etc. She is on the Board of Studies, Computer Engineering, Atmiya University, Rajkot. 